\title{
AN EFFICIENT INDUCTION MODULATOR FOR THE RELATIVISTIC KLYSTRON TWO BEAM ACCELERATOR*
}

\author{
S. R. Douglass ${ }^{\dagger}$, T. F. Godlove and E. Zaidman \\ FM Technologies, Inc., Fairfax, VA 22032, USA
}

\begin{abstract}
We describe design and testing of an induction cell with modulator for the Relativistic Klystron Two-Beam Accelerator (RK-TBA) concept, an option for the power source for the Next Linear Collider (NLC). FM Technologies has designed and built a prototype induction accelerator, which we call a re-accelerator, for a joint LLNL/LBNL TBA technology test-bed experiment.
\end{abstract}

\section{INTRODUCTION}

In the RK-TBA concept, microwave power $(11.4 \mathrm{GHz})$ is extracted for the NLC beam from a parallel relativistic, $600 \mathrm{~A}$ beam periodically along the beam line. The beam is bunched and relativistic $(10 \mathrm{MeV})$ so that the beam velocity is independent of its energy and it may keep up with the parallel travelling NLC beam being accelerated to GeV levels. Refs. [1], [2] have recent efforts on this.

Energy is restored to the high current beam using induction accelerators in between extraction points. Considering the power extracted and a $600 \mathrm{~A}$ beam, this translates to an accelerating gradient of $300 \mathrm{keV} / \mathrm{m}$. Also, in the original RK-TBA design, the accelerating potential has a $100 \mathrm{~ns}$ linear rise time and a $200 \mathrm{~ns}$ flat top. The pulse width is $300 \mathrm{~ns}$.

The FMT task is to produce an efficient prototype induction cell and modulator to drive it. We call our prototype a re-accelerator. See figure 1. The FMT reaccelerator consists of four parts:

1) The induction cell

2) The pulsed power modulator

3) The control chassis

4) The power supply

\section{INDUCTION CELL}

We have designed a half meter long induction cell that will provide $150 \mathrm{kV}$ across an accelerating gap. See figure 2. The $50 \mathrm{~cm}$ length is as long as practical, still allowing regular vacuum pump out sections, but reducing the total number of components to be connected and aligned.

\subsection{Core material and cell geometry}

When considering accelerator efficiency, core efficiency is the limiting factor, and therefore critical to the TBA and overall NLC efficiency. The core material is a Metglas alloy from Allied Signal, a high flux swing and low loss material. We use the 2605SC alloy, which has the greatest $\Delta \mathrm{B}$ swing and moderate losses per $\mathrm{m}^{3}$. The

* Supported by the DOE Small Business Innovation Research contract DE-FG02-95ER81972

†sdouglass@fm-technologies.com standard Metglas tape width is 2 in. so the module length can accommodate seven axial stacks of cores. We have a radial stack of two cores and they are referred to as the inner and outer cores. Two inner core locations are occupied by the induction gap, giving 12 cores, each driven with a 12.5 to $13 \mathrm{kV}$ pulse.

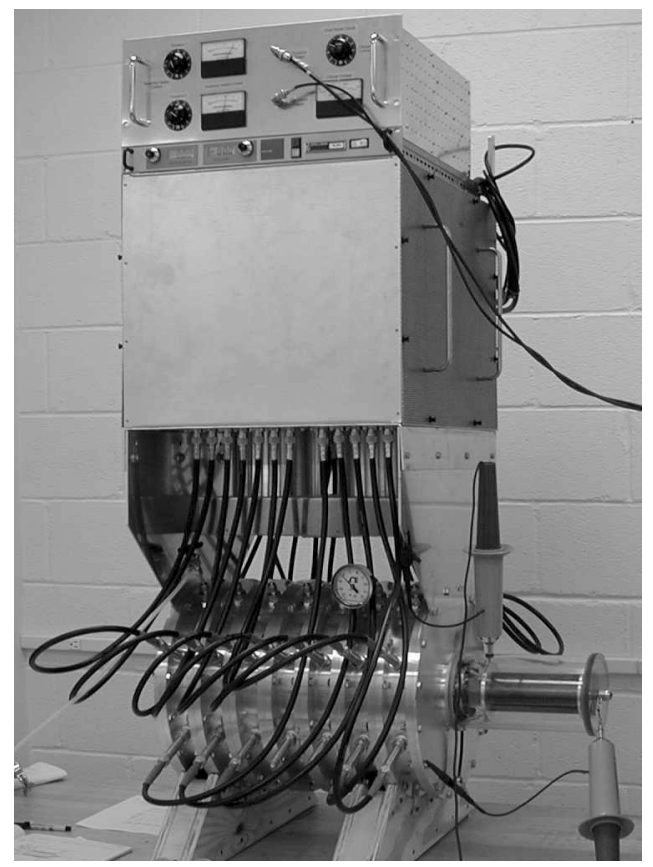

Figure 1: The FMT Re-accelerator

An inner and outer core are mounted together in a single unit with a shell containing high voltage feedthroughs and gas or oil ports for insulation and cooling. Seven of these units are stacked together and are capped off with endplates. The endplates center the vacuum beam pipe/gap assembly on axis and allow bolting to adjacent vacuum flanges. Finally, permanent magnet quadrupoles for beam transport are affixed to the outside of the beam pipe inside the cell.

\subsection{Induction Gap}

The design of the induction gap has many challenges of its own and is covered in another paper this conference. [3] The gap features low longitudinal impedance at the beam bunch frequency and high voltage hold off. The gap is integral with the beam transport pipe, but the gap/beam pipe is demountable from the induction cell, allowing for possible future upgrades in gap design. 


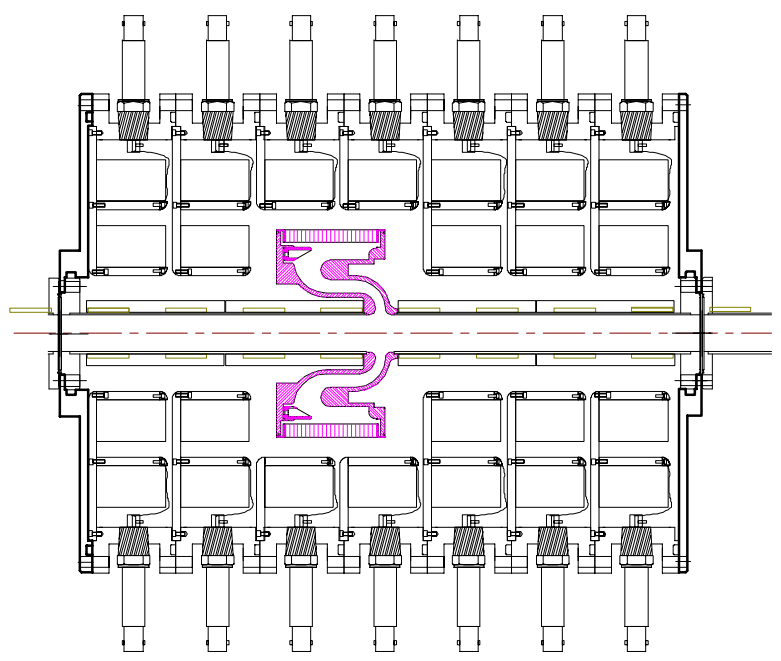

Figure 2: Diagram of Induction Cell

\subsection{Core Tests}

Core efficiency tests were performed by measuring, and taking the ratio of the output energy to the input energy of the core. The core under test was placed in an induction container with a resistively loaded secondary wire circuit on axis. The dummy resistor simulates the beam load as driven by a single core, i.e. $12.5 \mathrm{kV} / 600 \mathrm{~A}=20.8 \Omega$, the approximate resistance. Current in the secondary $\left(\mathrm{I}_{\mathrm{s}}\right)$ was measured with a Pearson current monitor. The core drive current from the modulator $\left(I_{p}\right)$ was also measured with a Pearson current monitor. The core drive voltage $\left(\mathrm{V}_{\text {gap }}\right)$ was measured as close to the induction gap as possible. The input power is defined as the product $\mathrm{V}_{\text {gap }} * \mathrm{I}_{\mathrm{p}}$ and the output power is defined as the product $\mathrm{V}_{\text {gap }} * \mathrm{I}_{\mathrm{s}}$. The power curves are integrated over the pulse width to find the energy transferred to the core and the portion of that energy transferred to the dummy load. The ratio of these two energies is the net efficiency of the core.

The inner cores have an inner radius of $5 \mathrm{~cm}$ and typically showed $60 \%$ efficiency. The outer cores have an inner radius of $10 \mathrm{~cm}$ and showed $47 \%$ efficiency. The total cell core efficiency is calculated to be $52 \%$ based on these figures. The typical energy loss for the material is 2400 to $2500 \mathrm{~J} / \mathrm{m}^{3}$, whether it is in an inner or outer core. Figure 3 shows the results of efficiency tests on four cores, two of the inner and two of the outer.

\section{MODULATOR}

The modulator contains 24 PFNs, twelve pairs. Each PFN output connects to its own cable. Each pair of cables drives opposite sides of a core.

\subsection{Pulse Forming Networks (PFNs)}

The PFNs are of two types, ones for the inner cores and ones for the outer cores. Each group of PFNs is switched by its own thyratron. PFN capacitors are rated for $30 \mathrm{kV}$. The inner core PFNs consist of four $2.0 \mathrm{nF}$ capacitors, each section containing a few turns of an inductor wrapped on a plastic bar. The outer core PFNs have four $2.5 \mathrm{nF}$ capacitors. The capacitors are attached by means of alligator clip leads. They handle the current well and the characteristic impedance can be modified to match the load. The load will vary when the beam current varies. The characteristic impedances can range a few ohms about $25 \Omega$ (inner) and $18 \Omega$ (outer). All PFNs have 0.5 $\mathrm{nF}$ capacitors across the outputs. This provides an initial linear rise in the pulse shape.

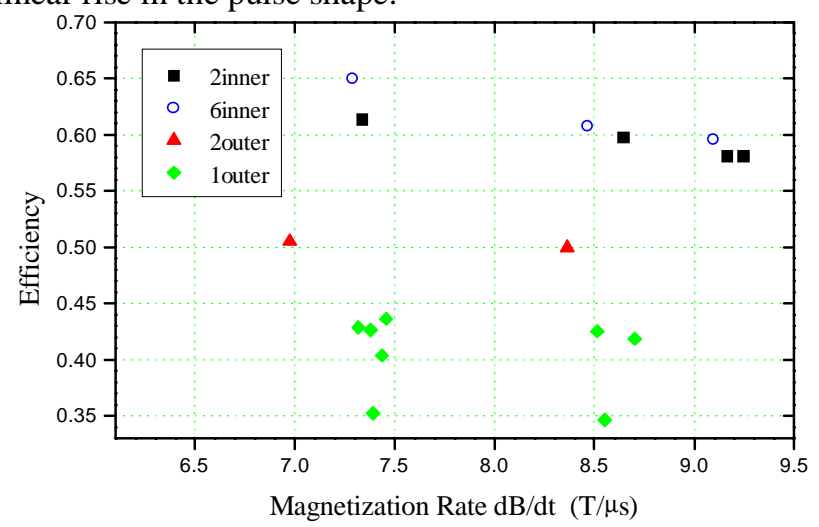

Figure 3. Efficiency for four cores. The different data points for each core correspond to different PFN configurations, core reset levels, and PFN charges.

\subsection{Thyratrons}

The modulator includes two 3 inch diameter, $35 \mathrm{kV}$ thyratrons (Triton F-130). There is one thyratron for the inner cores (5 kA peak current), and one for the outer cores (9.3 kA peak current). They handle up to $10 \mathrm{kA}$ of current for short pulses.

\section{CONTROL CHASSIS AND POWER SUPPLY}

The control chassis contains three major circuits:

1. Thyratron heaters

2. Thyratron triggers

3. Core reset pulsers

\subsection{Trigger Circuit}

The control chassis contains a printed circuit board that provides three functions:

1. A $-200 \mathrm{~V}$ bias for the control grids to prevent spurious triggering and aid in tube recovery to the open state.

2. An auxiliary pre-pulse to prime the tubes for fast switching.

3. A trigger pulse to be applied to the control grids of each tube.

The trigger pulse is split between two $35 \mathrm{~ns}$ delay lines. In the event one thyratron triggers first, the trigger will be loaded down, perhaps preventing the second thyratron from triggering. The delay lines provide $70 \mathrm{~ns}$ of isolation. The trigger signal is boosted by a 1:3 step up pulse transformer at the end of the delay line.

\subsection{Core Reset Circuit}

Core tests with d.c. reset currents reveal that the maximum flux swing is obtained with $5 \mathrm{~A}$ in inner cores 
and $15 \mathrm{~A}$ in outer cores. We use two pulsed circuits that can provide at least $25 \mathrm{~A}$ for 5 inner cores and $105 \mathrm{~A}$ for 7 outer cores. The two pulsed circuits are capacitors discharged into large inductors. The inductors create a slow discharge and also serve to isolate the reset circuit from the multi $\mathrm{kV}$ core pulse. There are several smaller parallel inductors in series with the main inductors. Their purpose is to isolate the outputs of the PFNs from each other. The thyratrons are triggered at the peak of the core reset discharge.

\subsection{Power Supply}

The power supply is from Spellman. It is an efficient switching supply with enough power to operate the modulator at a $1 \mathrm{~Hz}$ rate. It is current limited $(10 \mathrm{~mA}$ max.) to protect the thyratron in case it doesn't recover quickly so that current surges don't damage the tube. It linearly charges the PFNs to full voltage without the need for resonant doubling, avoiding losses in the large choke. Slow linear charging of the thyratron anode avoids capacitive coupling to the control grid and possible spurious triggering. It has. The power supply charges the PFNs through an $\mathrm{RC}$ filter $(\mathrm{RC}=7 \mu \mathrm{s})$ to protect the power supply.

\section{PERFORMANCE}

The re-accelerator is tested with dummy loads that are designed to simulate the beam impedance. The dummy load is $20.8 \Omega$ per core, $250 \Omega$ for the full cell. The full cell load is designed to handle $150 \mathrm{kV}$ It provides for voltage division so that the peak voltage can be measured. The cell was tested without the induction gap installed. The design is complete, but it is still under construction.

\subsection{Single Core Pulse}

Figure 4 shows a full $12.5 \mathrm{kV}$ pulse on one outer core. Proper PFN tuning has allowed a 100 ns linear rise and a flat top. However, the flat top is less that the desired 200 ns because the maximum flux swing of our 2605SC material is $2.4 \mathrm{~T}$, less than the $3.0 \mathrm{~T}$ specification.

In this example, a $20 \mathrm{nF}$ PFN charged to $26 \mathrm{kV}$ was used to create this pulse. The $1 / 2 \mathrm{CV}^{2}$ stored energy is 6.76 Joules. The energy transferred to the core in the initial pulse is 3.46 Joules (51\%). The rest is dissipated in post pulse ringing of the core. The energy in the load is 1.91 Joules, giving a core efficiency of $55 \%$. The total reaccelerator efficiency is $28 \%$. The modulator is relatively inefficient because the PFN is tapered for a long pulse in an attempt to extend the flat top. However, if the PFN is matched to the natural pulse width of the core, then modulator efficiencies greater than $90 \%$ are possible.

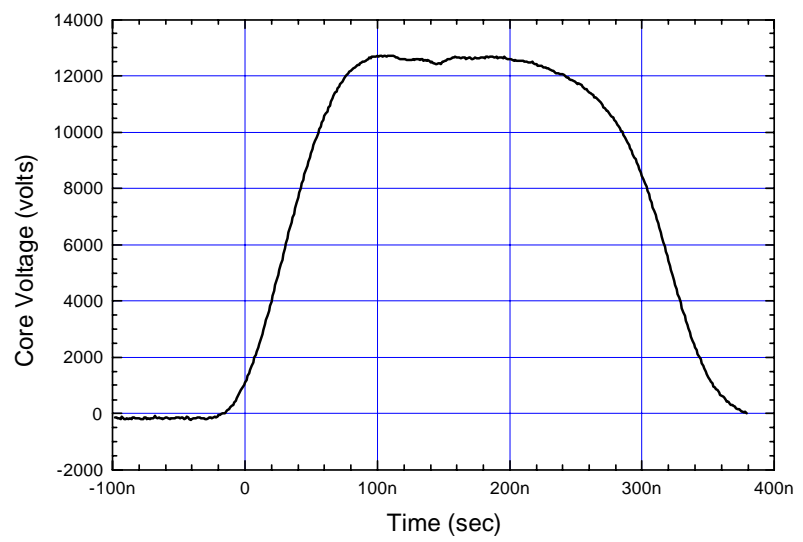

Figure 4. Core pulse on one outer core.

\subsection{Full Module Pulse}

Figure 5 shows the full re-accelerator pulse that we have achieved so far. Adjustments to the dummy load are required, as well as tuning of the PFNs. The full modulator PFN capacitance is $220 \mathrm{nF}$. The modulator was charged to $21 \mathrm{kV}$ for the figure 5 pulse. The stored energy is 48.5 Joules. The energy in the load in the initial pulse is 25.5 Joules. The re-accelerator efficiency is $52.6 \%$. The modulator efficiency was not determined independently, however, the core efficiency is about $52 \%$. This means the modulator must be very close to $100 \%$ efficient.

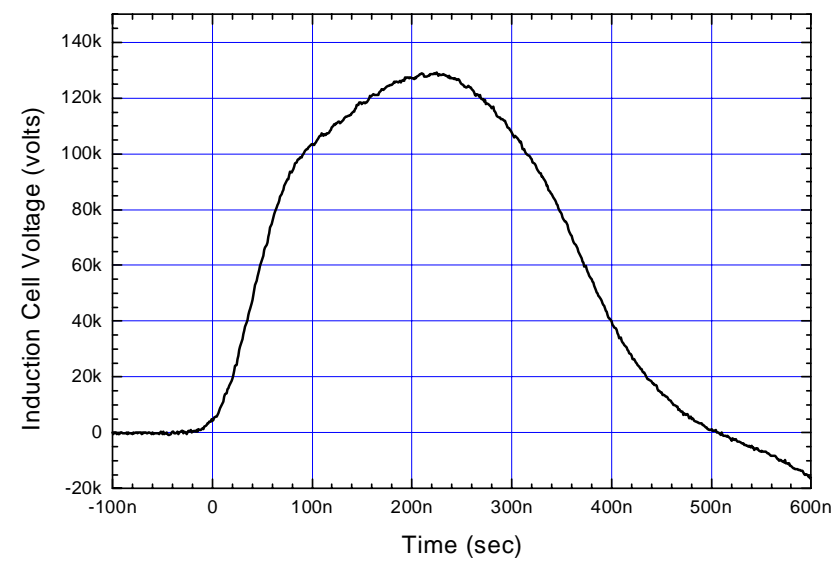

Figure 5. The maximum voltage pulse produced by the reaccelerator thus far.

\section{REFERENCES}

[1] Timothy Houck, "Beam Dynamics Experiments in Support of Relativistic Klystrons", PAC'01, Chicago, June 2001.

[2] Steve Lidia, "RF Systems for the Betatron Node Scheme Experiment at LBNL", PAC'01, Chicago, June 2001.

[3] E. Zaidman, S. Douglass, and T. Godlove, "Acceleration Gap for the Relativistic-Klystron TwoBeam Accelerator', PAC'01, Chicago, June 2001. 